\title{
Divergência fenotípica entre acessos de uvas de mesa no Semi-Árido brasileiro
}

\author{
Rita Mércia Estigarribia Borges ${ }^{(1)}$, Nadja Pollyanna da Silva Gonçalves ${ }^{(1)}$, Ana Patrícia de Oliveira Gomes ${ }^{(1)}$ \\ e Elaini Oliveira dos Santos Alves ${ }^{(2)}$
}

(1)Embrapa Semi-árido, Caixa Postal 23, CEP 56300-970 Petrolina, PE. E-mail: rmborges@cpatsa.embrapa.br, nadja.pollyanna@cpatsa.embrapa.br, apgomes@cpatsa.embrapa.br ${ }^{(2)}$ Universidade Estadual de Santa Cruz, Departamento de Genética, Campus Soane Nazaré de Andrade, Km 16, Rodovia Ilhéus-Itabuna, CEP 45662-000 Ilhéus, BA. E-mail: elaini@cpatsa.embrapa.br

\begin{abstract}
Resumo - O objetivo deste trabalho foi avaliar a divergência fenotípica em uvas da Coleção da Embrapa Semi-Árido, com base em oito variáveis morfoagronômicas avaliadas nos anos 2005 e 2006. Nas uvas com sementes, a análise por componentes principais identificou oito grupos contrastantes. As variáveis mais relevantes, quanto à divergência genética, foram peso e comprimento de bagas. Em uvas sem sementes, formouse apenas um grupo, e as variáveis mais relevantes foram peso de bagas e produção. A distância euclidiana média padronizada no grupo com sementes mostrou que $41 \%$ dos acessos apresentaram estimativas acima da média geral. No grupo sem sementes, o percentual foi de $47 \%$. Foi observada concordância de resultados com os componentes principais. Pelo UPGMA, formaram-se três grandes grupos das uvas com sementes: o primeiro, com 14 acessos; o segundo, com seis acessos e similaridade de 56\%; e o terceiro, com 19 acessos e similaridade de $73 \%$. Das uvas sem sementes, formaram-se três grupos: um, com 11 acessos e similaridade de $59 \%$; outro, com sete acessos e similaridade de $58 \%$; e o terceiro com apenas um acesso. Sugere-se que os acessos com maior divergência quanto aos caracteres estudados sejam utilizados para a obtenção de híbridos de videira.
\end{abstract}

Termos para indexação: Vitis vinifera, descritores de videira, divergência genética, melhoramento genético.

\section{Phenotypic divergence among table grapes accesses in the Brazilian Semi-Arid}

\begin{abstract}
The aim of this work was to evaluate the phenotypic divergence in grapes from the Collection of Embrapa Semi-Árido, in 2005/2006, based on eight morphoagronomic variables. In seeded grapes, the analysis by main components identified eight contrasting groups. The most important variables for genetic divergence were weight and length of berries. In seedless grapes, only one group was found, with berry weight and yield as the most important variables. The standardized mean Euclidean distance in the seeded grapes showed that $41 \%$ accesses had estimative higher than the general mean; in the seedless grapes, it was $47 \%$. Agreement with the principal components was observed. Through UPGMA, three big groups with seeds were formed: the first with 14 accesses; the second with six accesses and 56\% similarity; the third with 19 accesses and $73 \%$ similarity. In the seedless group, two groups were formed: one with 11 accesses and 59\% similarity; another with seven accesses and 58\% similarity; and the third with one access. It is suggested that accesses with higher divergence for the studied characters be used to obtain grape hybrids.
\end{abstract}

Index terms: Vitis vinifera, grape descriptors, genetic divergence, genetic inbreeding.

\section{Introdução}

Os recursos genéticos apresentam valor inestimável para o melhoramento de uma determinada espécie vegetal (Nass, 2001). No manejo de coleções, estimar a divergência fenotípica entre acessos significa estabelecer estratégias para a organização das informações que irão otimizar o uso do germoplasma, em programas de melhoramento (Carvalho et al., 2003; Moura, 2003; Sudré et al., 2005; Oliveira et al., 2007). Segundo Oliveira et al. (2003), a utilização de técnicas multivariadas para estimar a divergência genética tem-se tornado comum entre os melhoristas de plantas. Os autores ainda citam que, dessas técnicas, as mais empregadas são: a análise por componentes principais, quando os dados são obtidos de experimentos sem repetições; e os métodos de agrupamento, cuja aplicação depende da utilização de uma medida de dissimilaridade previamente estimada.

Segundo This et al. (2006), nos últimos anos, em relação à videira, é cada vez mais crescente o interesse na busca de informações sobre a origem da espécie 
Vitis vinifera, bem como a busca da diversidade nela existente. Os autores relatam a importância da análise de dados morfológicos para a determinação da variabilidade em videira, pela qual a caracterização e a avaliação morfoagronômica de germoplasma vêm sendo feitas para identificação de diversidade dentro e entre espécies. Ainda, com a mesma espécie, vários trabalhos foram feitos com a utilização de descritores morfológicos para estudos de divergência (Martínez et al., 2003; Coelho et al., 2004; This et al., 2006).

A videira para o consumo in natura, no Vale do Submédio São Francisco, tornou-se o agronegócio mais importante da região, responsável pela geração de empregos e divisas no Semi-Árido brasileiro. Em razão disto, a Embrapa Semi-Árido implantou uma coleção de trabalho, para dar suporte ao melhoramento da espécie quanto à adaptabilidade dos acessos às condições locais.

O objetivo deste trabalho foi analisar a diversidade fenotípica entre 58 acessos de videira, com e sem sementes, da Coleção da Embrapa Semi-Árido, com base em oito variáveis quantitativas.

\section{Material e Métodos}

O trabalho foi realizado em 58 acessos da coleção de videira da Embrapa Semi-Árido, no Campo Experimental de Mandacaru, Município de Juazeiro, BA, a $9^{\circ} 24^{\prime} \mathrm{S}, 40^{\circ} 26^{\prime} \mathrm{W}$ e $375 \mathrm{~m}$ de altitude, em clima tropical quente e seco. A temperatura média anual é de $23,6^{\circ} \mathrm{C}$, a umidade relativa do ar é de $67,1 \%$ e a precipitação média anual é de $455 \mathrm{~mm}$. Os solos predominantes no local são classificados como Vertissolos (Embrapa, 1999).

As estimativas das dissimilaridades fenotípicas foram obtidas de acessos com e sem sementes (Tabela 1), em quatro ciclos de cultivo, em 2005 e 2006, pela avaliação das quatro plantas de cada acesso. Foram utilizados oito caracteres: produção média das plantas (g); média do número de cachos das quatro plantas; peso médio de cinco cachos (g), obtido pela média de cinco cachos por planta, coletados ao acaso; comprimento médio dos cachos $(\mathrm{cm})$; diâmetro médio dos cachos $(\mathrm{cm})$; peso médio de bagas $(\mathrm{g})$, obtido pela média de dez bagas por cacho, coletadas ao acaso; comprimento médio de bagas $(\mathrm{cm})$, obtido pela média de dez bagas por cacho, coletadas ao acaso; diâmetro médio de bagas $(\mathrm{cm})$, obtido pela média de dez bagas por cacho, coletadas ao acaso.
Para obtenção das estimativas, utilizaram-se: a análise da divergência genética por componentes principais e distância euclidiana média, calculada com os dados obtidos, padronizados com base nos oito descritores selecionados indicados por Cruz \& Regazzi (1994) para acessos instalados sem casualização e controle local. Para a análise dos dados, utilizou-se o programa GENES (Cruz, 2006).

As matrizes simétricas geradas pelas distâncias entre os pares de acessos foram utilizadas para a formação dos agrupamentos, tendo-se empregado o método hierárquico aglomerativo de ligação média não padronizada (UPGMA), e as dissimilaridades foram identificadas em dendogramas dos dois grupos de acessos estudados, com e sem sementes.

\section{Resultados e Discussão}

A divergência fenotípica entre os 39 acessos do grupo de uvas com sementes e os 19 acessos do grupo de uvas sem sementes, obtida pela análise de componentes principais, está representada na Tabela 2 e Figura 1, e a obtida por dissimilaridades mínima e máxima e por fenograma, na Figura 2.

$\mathrm{Na}$ análise dos acessos de uvas com sementes, evidenciou-se que os dois primeiros componentes explicaram a variação total disponível (Tabela 2), condição que permite a análise da divergência por meio da dispersão gráfica (Cruz \& Regazzi, 1994). Observouse a formação de oito grupos contrastantes de acessos, e os acessos Muscat Caillaba, Seyve Villard 20365 e Branca Salitre estão localizados isoladamente dos grupos formados (Figura $1 \mathrm{~A}$ ). A variável que mais contribuiu para a divergência genética foi o peso médio de bagas, com $56,96 \%$ de relevância para a variabilidade entre os acessos, e a seguir, o comprimento da baga, com 23,38\% (Tabela 2).

$\mathrm{Na}$ análise dos acessos de uvas sem sementes, os dois primeiros componentes explicaram $67,80 \%$ da variação total disponível (Tabela 2). $\mathrm{Na}$ análise da dispersão gráfica, formou-se um grupo que continha a minoria dos acessos (Figura $1 \mathrm{~B}$ ). As variáveis que mais contribuíram para a divergência genética foram o peso médio de bagas e a produção, com 46,24 e 21,56\% de contribuição para a variabilidade entre os acessos, respectivamente (Tabela 2).

Pelo método da distância euclidiana média, no grupo com sementes, as dissimilaridades foram mínima de 0,10761, nos acessos CG 4113 e CG 33716, e máxima 
de 2,679505, nos acessos CG 33716 e Branca Salitre. Os dois últimos são, portanto, os acessos de maior divergência. Branca Salitre foi um acesso coletado na região do Salitre (Juazeiro, BA) e que necessita de estudos moleculares para sua correta identificação. Ao se analisarem comparativamente a dispersão gráfica, em componentes principais, e os valores máximos de dissimilaridade, por meio da distância euclidiana média, foi possível observar a concordância de resultados, uma vez que os acessos mais dispersos coincidem com os de CG 4113 e Branca Salitre (Figura 1 A). A média geral das estimativas $\left(\operatorname{dg}_{\mathrm{E}}\right)$ foi de 1,318044 . Dos 39 acessos estudados, 16 (41\%) apresentaram médias das estimativas acima da média geral. São eles:
Panse Precoce, Stover, CG 4113, CG 39915, Moscatel Nazareno, CG 33716, CG 38049, Red Globe, Rosaki Rosado, Dominga, Seyve Villard 20365, CG 90450, Soraia, Seyve Villard 12375, Estevão Marinho e Branca Salitre.

No grupo de uvas sem sementes, os valores foram: mínimo de 0,353506, nos acessos Júpiter e Thompson Seedless; e máximo de 2,777203, nos acessos Saturn e BRS Linda. Os valores máximos também foram concordantes com os acessos de maior dispersão, pela análise dos componentes principais (Figura $1 \mathrm{~B}$ ), em que as maiores dissimilaridades foram observadas em Saturn e Marro Seedless e Saturn e BRS Linda, respectivamente (Figura $1 \mathrm{~B}) . \mathrm{A} \mathrm{dg}_{\mathrm{E}}$ teve valor

Tabela 1. Identificação de acessos da coleção de videira da Embrapa Semi-Árido (2007).

\begin{tabular}{|c|c|c|c|}
\hline \multirow[t]{2}{*}{ Identificação do acesso } & Parentais & Identificação do acesso & Parentais \\
\hline & \multicolumn{2}{|c|}{ Genótipos com sementes } & \\
\hline \multirow[t]{2}{*}{ 1. H44969 } & \multirow[t]{2}{*}{ Fernão Pires x Pirovano 75} & \multirow[t]{2}{*}{ 21. Juliana } & Itália (Bicane x Moscatel de Hamburgo) \\
\hline & & & x Madalena \\
\hline 2. Kagina & $\mathrm{PNI}^{(1)}$ & 22. Red Globe & (Emperor x Hunisa) x Nocera \\
\hline 3. Cardinal & Flame Tokay x Alfonso Lavallée & 23. Orange Muscat & $\mathrm{PNI}^{(1)}$ \\
\hline 4. Panse Precoce & $\mathrm{PD}^{(2)}$ & 24. Sovrana Pirovano & Frankenthal x Delizia de Vaprio \\
\hline 5. Dona Maria & Moscatel de Alexandria x Rosaky & 25. Ferral & $\mathrm{PNI}^{(1)}$ \\
\hline 6. Moscatel Branco & $\mathrm{PNI}^{(1)}$ & 26. IAC 77526 & $\mathrm{PNI}^{(1)}$ \\
\hline 7. Stover & $\mathrm{PD}^{(2)}$ & 27. Rosaki Rosado & $\mathrm{PD}^{(2)}$ \\
\hline 8. CG40016 & Moscatel Rosado x Sultanina & 28. Dominga & $\mathrm{PD}^{(2)}$ \\
\hline 9. Niágara Rosada & $\begin{array}{l}\text { Mutação natural da Niágara Branca (Vitis } \\
\text { labrusca x Vitis vinifera) }\end{array}$ & 29. Seyve Villard 20365 & $\mathrm{PNI}^{(1)}$ \\
\hline 10. Moscato Noir & $\mathrm{PNI}^{(1)}$ & 30. Dattier de Beyrouth & $\mathrm{PD}^{(2)}$ \\
\hline 11. Muscat Caillaba & $\mathrm{PD}^{(2)}$ & 31. CG 90450 & Molinera Gorda x Cardinal \\
\hline 12. Piratininga & Mutação somática da variedade IAC 8424 & 32. Soraia & Higland x Golden Queen \\
\hline 13. CG 4113 & Lambrusco x Carignane & 33. Moscatel de Hamburgo & Moscatel de Alexandria x Frankenthal \\
\hline 14. H449100 & Fernão Pires x Pirovano 75 & 34. Emerald & $\mathrm{PNI}^{(1)}$ \\
\hline 15. CG 39915 & Saint Jeannet x Sultanina & 35. Seyve Villard 12375 & Seibel $6468 \times 6905$ \\
\hline 16. Frankenthal & $\mathrm{PD}^{(2)}$ & 36. Baresana & $\begin{array}{l}\text { Golden Hamburg x Muscatel de } \\
\text { Alexandria }\end{array}$ \\
\hline \multicolumn{2}{|c|}{ 17. Moscatel Nazareno Moscatel de Hamburgo x João Santarém } & 37. Império & $\mathrm{PNI}^{(1)}$ \\
\hline 18. CG33716 & Dattier de Beyrouth x Sultanina & 38. Estevão Marinho & $\mathrm{PD}^{(2)}$ \\
\hline 19. CG 38049 & $\begin{array}{l}\text { Riesling Rhoda x CG377 (Gibi x } \\
\text { Sultanina) }\end{array}$ & 39. Branca Salitre & $\mathrm{PD}^{(2)}$ \\
\hline 20. Ferlongo & $\mathrm{PNI}^{(1)}$ & & \\
\hline \multicolumn{4}{|c|}{ Genótipos sem sementes } \\
\hline 1. Adona & Soraia x IAC 544-14 & 11. Marroo Seedless & Carolina Blackrose x Ruby Seedless \\
\hline 2. Paulistinha & Niágara Branca x Sultanina & 12. CG 87908 & Moscatel Rosado $\mathrm{n}^{\mathrm{o}} 2 \mathrm{x}$ Beauty Seedless \\
\hline 3. Vênus & Alden x NY 46000 & 13. Fiesta & $\mathrm{PNI}^{(1)}$ \\
\hline 4. Beauty Seedless & Queen of the Vineyard $x$ Black Kishmish & 14. Thompson Seedless & $\mathrm{PD}^{(2)}$ \\
\hline 5. Júpiter & $\begin{array}{l}\text { Gold x Glenora (Ontário x Russian } \\
\text { Seedless) }\end{array}$ & 15. BRS Morena & Marroo Seedless x Centennial \\
\hline 6. Flame Seedless & $\begin{array}{l}\text { (Cardinal x Sultanina) x (Red Málaga x } \\
\text { Tifanini Ahmer) x (Málaga x Sultanina) }\end{array}$ & 16. BRS Linda & CNPUV 154-90 x Saturn \\
\hline 7. Dawn Seedless & Gold x Perlette & 17. CG 87746 & Moscatel Rosado $n^{0} 2 \times$ Beauty Seedless \\
\hline 8. Saturn & $\mathrm{PNI}^{(1)}$ & 18. Sutanina Branca & $\mathrm{PD}^{(2)}$ \\
\hline $\begin{array}{l}\text { 9. Loose Perlette } \\
\text { 10. Neptune }\end{array}$ & $\begin{array}{l}\text { Muscat Reine des Vignes x Sultanina } \\
\text { PNI }^{(1)}\end{array}$ & 19. BRS Clara & CNPUV 154-147 x Centennial \\
\hline
\end{tabular}

\footnotetext{
(1) Parental não identificado. ${ }^{(2)}$ Parental desconhecido.
} 
correspondente a 1,332262 e percentual de $47 \%$ de acessos com média acima da geral, correspondentes aos acessos: Adona, Paulistinha, Flame Seedless, Saturn, Loose Perlette, Neptune, Marro Seedless, BRS Morena e BRS Linda.

Costa et al. (2006) salientam que estudos relacionados à divergência genética fornecem parâmetros para a identificação de genitores favoráveis à obtenção de populações segregantes, em programas de hibridação. A determinação de altos percentuais de acessos divergentes, dentro do germoplasma estudado, é importante para programas de hibridação, levandose em conta as características analisadas. Os autores ressaltam que, em caso de divergência acentuada, tal informação é útil para se restringir o número de genitores a ser escolhido. No caso da coleção de videira, o método indica a necessidade do aumento da variabilidade quanto às variáveis analisadas.

No que se refere à análise de agrupamento, obtida por meio das estimativas de dissimilaridade, em relação ao grupo com sementes, observou-se a formação de três grandes grupos: o primeiro foi composto pelos acessos Frankenthal, Branca Salitre, Baresana, Império, H449100, CG 38049, Emerald, IAC 77526, Muscat Caillaba, Stover, Juliana, CG 33716, Red Globe e Maria (Figura 2 A); o segundo foi constituído por Niágara Rosada, Ferral, CG 39915, Seyve Villard 20365, Moscatel Branco e CG 4113. O nível de similaridade do grupo foi de $56 \%$; o terceiro grupo foi o que apresentou maior dissimilaridade fenotípica com $73 \%$ (Figura $2 \mathrm{~A}$ ), composto por Orange Muscat, Estevão Marinho, Moscatel Nazareno, Seyve Villard 12375, Dattier de Beyrouth, Moscato Noir, Cardinal, CG 40016, H449100, Panse Precoce, CG 90450, Piratininga, Ferlongo, Dominga, Sovrana

Tabela 2. Estimativa dos autovalores em acessos de videira no Semi-Árido brasileiro em 2007.

\begin{tabular}{rrrrr}
\hline \multicolumn{2}{c}{ Acessos com sementes } & & \multicolumn{2}{c}{ Acessos sem sementes } \\
\cline { 1 - 2 } \cline { 5 - 5 } Raiz (\%) & $\begin{array}{c}\text { Percentagem } \\
\text { acumulada }\end{array}$ & & Raiz $(\%)$ & $\begin{array}{c}\text { Percentagem } \\
\text { acumulada }\end{array}$ \\
\hline 56,96 & 56,96 & & 46,24 & 46,24 \\
23,38 & 80,35 & & 24,56 & 67,80 \\
8,34 & 88,69 & & 18,21 & 86,02 \\
5,28 & 93,97 & & 6,83 & 92,85 \\
2,92 & 96,90 & & 4,56 & 97,41 \\
1,36 & 98,26 & & 2,08 & 99,50 \\
1,20 & 99,47 & & 0,39 & 99,90 \\
0,52 & 100,00 & & 0,99 & 100,00 \\
\hline
\end{tabular}

Pirovano, Soraia, Kagina, Rosaki Rosado e Moscatel de Hamburgo. Na avaliação dos três grandes grupos, foi possível observar as dissimilaridades entre seis acessos denominados CG's (CG 40016, CG 4113, CG 39915, CG 33716, CG 38049 e CG 90450), que se distribuíram em subgrupos diferentes. No primeiro grande grupo, o grau de similaridade entre os acessos CG 38049 e CG 33716 foi de 57\%, semelhante ao acesso Ferral (Figura 2 A). No segundo grupo, os acessos CG 39915 e CG 4113 foram similares em $19 \%$, e o primeiro foi mais similar ao Moscatel Branco (Figura 2 A). No grupo três, a similaridade entre os CG's foi de $15 \%$ (Figura $2 \mathrm{~A}$ ).

No grupo sem sementes, foi observada a formação de três grupos principais: o primeiro foi composto por
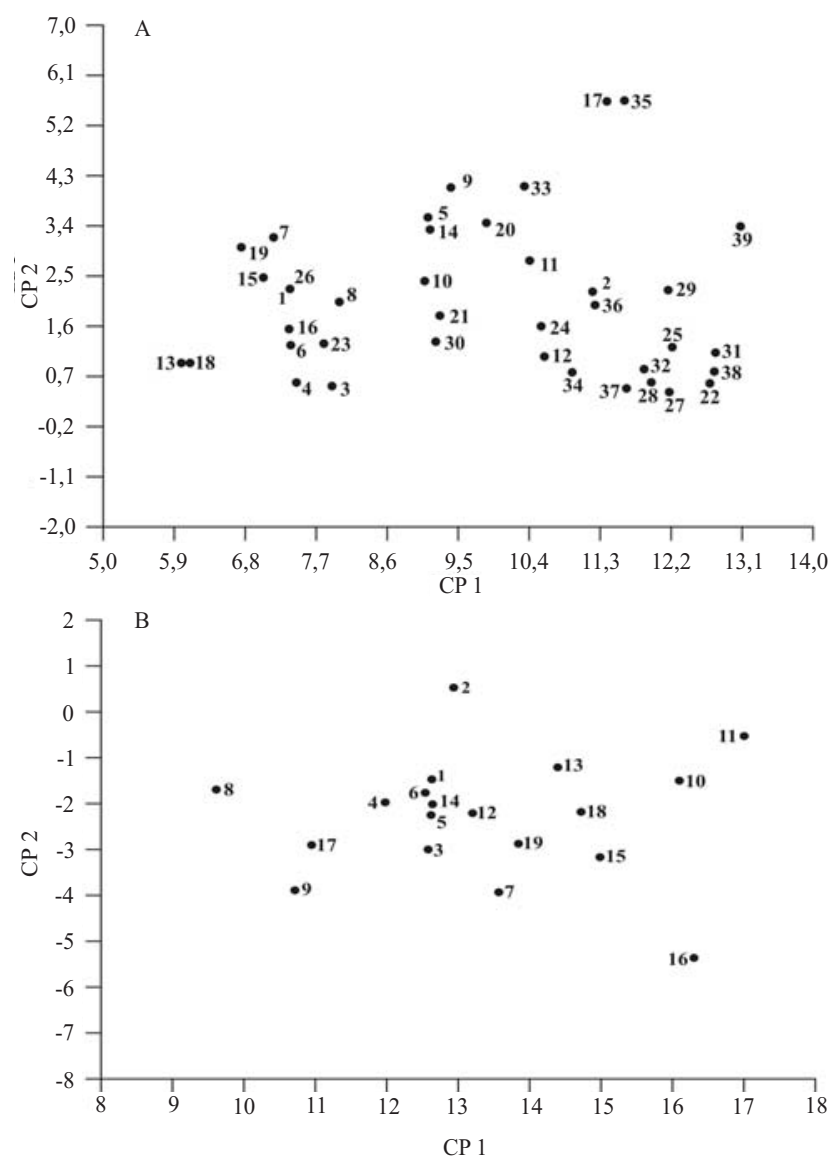

Figura 1. Dispersão gráfica gerada a partir dos escores dos componentes (CP) 1 e 2, na análise de componentes principais dos acessos com sementes (A) e sem sementes (B). Identificação dos genótipos conforme Tabela 1. 
dois subgrupos com similaridade de 59\% (Figura 2 B), formado por 11 acessos - Flame Seedless, BRS Clara, Neptune, Fiesta, Beauty Seedless, Marroo Seedless, Saturn, CG 87746, CG 87908, Thompson Seedless e Paulistinha; no segundo, a similaridade entre os acessos foi em torno de $58 \%$, e foi composto por sete acessos - Adona, Júpiter, Sultanina Branca, BRS Morena, BRS Linda, Dawn Seedless e Loose Perlette; o terceiro grupo foi o que apresentou similaridade total, porém composto somente pelo acesso Vênus (Figura 2 B).

Em relação à genealogia, no grupo com sementes, observou-se que os acessos CG 38049 e CG 33716 estão no mesmo agrupamento e têm um parental em comum, a cultivar Sultanina (Tabela 1). O mesmo fato foi observado em 'Baresana' e 'Maria', que têm 'Moscatel de Alexandria' como um dos parentais (Tabela 1). Os acessos H44969
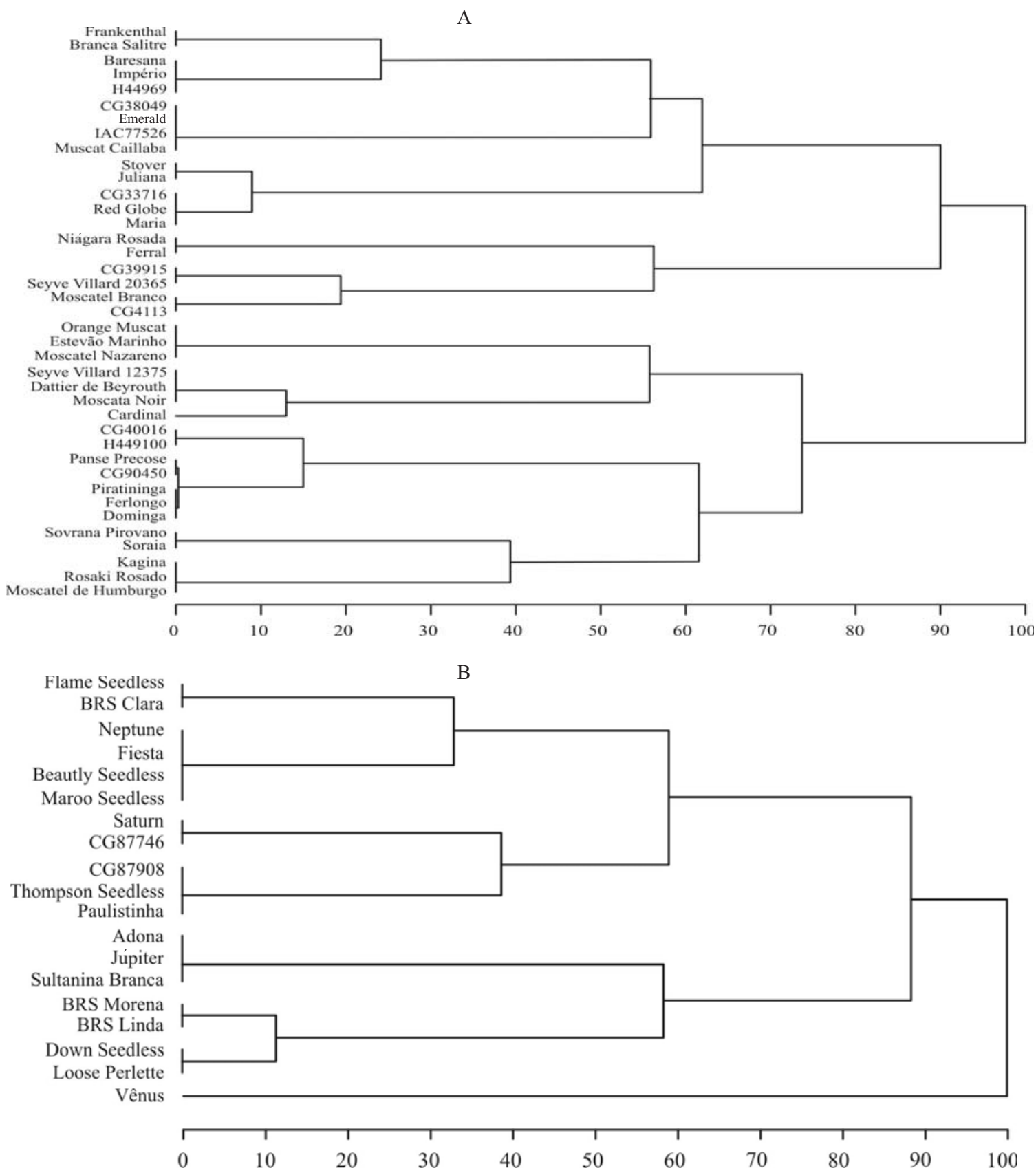

Figura 2. Fenograma gerado pelo método UPGMA, a partir das dissimilaridades fenotípicas, em 39 acessos de uvas com sementes (A) e em 19 acessos de uvas sem sementes (B). 
e H449100, embora apresentem a mesma genealogia, encontram-se em grupos distintos, provavelmente porque são híbridos superiores, resultantes de seleção de um mesmo cruzamento. Nos acessos sem sementes, observouse que BRS Clara e BRS Linda, apesar de apresentarem a mesma genealogia (Tabela 1), encontram-se em grupos distintos (Figura 2 A). Nos demais acessos, com e sem sementes, de genealogia conhecida, não foi verificado nenhum grau de parentesco.

Em relação aos resultados obtidos, foi possível observar que, no grupo com sementes, o fenograma identificou maiores níveis de dissimilaridade, destacados o primeiro e o terceiro grupos com $61 \%$.

Segundo Dias (1994), a postulação de critérios racionais e eficientes para a identificação de tipos parentais, para a síntese de híbridos em programas de melhoramento, é promissora quando se utilizam métodos de identificação de alto grau de divergência, o que significa economia de recursos financeiros, de tempo e de mão-de-obra. A identificação dos grupos influenciará positivamente na escolha dos acessos para a realização dos cruzamentos, de forma a se priorizarem os acessos, nos grupos de uvas com e sem sementes analisados, que apresentem maior divergência. Porém, vale ressaltar que os resultados obtidos pela análise de dissimilaridades dos coeficientes mínimo e máximo (Tabela 2) demonstram a necessidade do aumento da variabilidade dentro da coleção. Martínez et al. (2003), ao estudar a diversidade em variedades de videira cultivadas na Argentina, relataram a importância da análise de descritores morfoagronômicos. Nas variedades estudadas, os autores encontraram a formação de três grupos distintos, dentro da espécie Vitis vinifera, o que complementou os estudos com marcadores moleculares, que funcionaram como importante ferramenta para a identificação de maiores graus de diversidade nas variedades cultivadas na Argentina.

\section{Conclusões}

1. Háníveis médios de divergência fenotípica na coleção de videira da Embrapa Semi-Árido, quanto aos caracteres avaliados, principalmente nos acessos com sementes.

2. Há concordância de resultados, na indicação de maior divergência entre os acessos com sementes (CG 33716 e Branca Salitre) e sem sementes (Saturn e BRS Linda), tanto na análise por componentes principais quanto por distância euclidiana média.

\section{Referências}

CARVALHO, S.I.C. de; BIANCHETTI, L. de B.; BUSTAMANTE, P.G.; SILVA, D.B. da. Catálogo de germoplasma de pimentas e pimentões (Capsicum spp.) da Embrapa Hortaliças. Brasília: Embrapa Hortaliças, 2003. 49p. (Embrapa Hortaliças. Documentos, 49).

COELHO, I.; CUNHA, J.; CUNHA, J.P.; CARNEIRO, L.C.; CASTRO, R.; EIRAS DIAS, J.E. Comparação ampelométrica de populações selvagens de Vitis vinifera L. e de castas antigas do Sul de Portugal. Ciência e Técnica Vitivinícola, v.19, p.1-12, 2004.

COSTA, M.N. da; PEREIRA, W.E.; BRUNO, R. de L.A.; FREIRE, E.C.; NÓBREGA, M.B. de M.; MILANI, M.; OLIVEIRA, A.P. de. Divergência genética entre acessos e cultivares de mamoneira por meio de estatística multivariada. Pesquisa Agropecuária Brasileira, v.41, p.1617-1622, 2006.

CRUZ, C.D. Programa GENES: análise e processamento de dados baseado em modelos biométricos e em estatística experimental. Viçosa: UFV, 2006. 1 CD-ROM.

CRUZ, C.D.; REGAZZI, A.J. Diversidade genética. In: CRUZ, C.D.; REGAZZI, A.J. Modelos biométricos aplicados ao melhoramento genético. Viçosa: UFV, 1994. p.287-313.

DIAS, L.S.A. Divergência genética e fenética multivariada na predição de híbridos e preservação de germoplasma de cacau (Theobroma cacao L.). 1994. 94p. Tese (Doutorado) - Escola Superior de Agricultura Luiz de Queiroz, Piracicaba.

EMBRAPA. Sistema brasileiro de classificação de solos. Brasília: Embrapa-SPI; Rio de Janeiro: Embrapa-CNPS, 1999. 412p.

MARTÍNEZ, L.; CAVAGNARO, P.; MASUELLI, R.; RODRÍGUEZ, $\mathrm{J}$. Evaluation of diversity among Argentine grapevine (Vitis vinifera L.) varieties using morphological data and AFLP markers. Electronic Journal of Biotechnology, v.6, p.242-250, 2003.

MOURA, E.F. Divergência genética entre acessos de jaborandi (Pilocarpus microphyllus). 2003. 75p. Dissertação (Mestrado) Universidade Federal de Lavras, Lavras.

NASS, L.L. Utilização de recursos genéticos vegetais no melhoramento. In: NASS, L.L.; VALOIS, A.C.C.; MELO, I.S. de; VALADARES-INGLIS, M.C. Recursos genéticos e melhoramento de plantas. Rondonóplis: Fundação MT, 2001. p.29-56.

OLIVEIRA, F.J. de; ANUNCIAÇÃO FILHO, C.J. da; BASTOS, G.Q.; REIS, O.V. dos. Divergência genética entre cultivares de caupi. Pesquisa Agropecuária Brasileira, v.38, p.605-611, 2003.

OLIVEIRA, M. do S.P. de; FERREIRA, D.F.; SANTOS, J.B. dos. Divergência genética entre acessos de açaizeiro fundamentada em descritores morfoagronômicos. Pesquisa Agropecuária Brasileira, v.42, p.501-506, 2007.

SUDRÉ, C.P.; RODRIGUES, R.; RIVA, E.M.; KARASAWA, M.; AMARAL JÚNIOR, A.T. do. Divergência genética entre acessos de pimenta e pimentão utilizando técnicas multivariadas. Horticultura Brasileira, v.23, p.22-27, 2005.

THIS, P.; LACOMBE, T.; THOMAS, M.R. Historical origins and genetic diversity of wine grapes. Trends in Genetics, v.22, p.511-519, 2006. 